\title{
Pulse Packing in Magnetic Recording Wire ${ }^{1}$
}

\author{
By Irvin L. Cooter
}

\begin{abstract}
An oscillographic method is described for determining the relative pulse packing of different types of magnetic recording wire used for pulse storage. Typical curves are presented to show the influence of the amplitude, duration, and repetition rate of the magnetizing pulses on the pulse packing in two types of recording wire.

A powder pattern method is also described for visually observing the length of the magnets produced in the wire by the magnetizing pulses. Photographs are shown of typical patterns. The data obtained by this method verify the pulse packing data obtained by the oscillographic method.
\end{abstract}

\section{Introduction}

One of the principal requirements of high-speed electronic digital computers is the rapid interchange of data between their input and output components. It is also of advantage to be able to erase and correct recorded data or to discard a given set of data and use the same storage medium again for another set. The ease with which the magnetization of a wire or tape can be erased has led to the adoption of magnetic recording for this purpose. In this application, information in the form of coded groups of magnetic pulses is stored in a magnetic wire or tape, and after this information has served its purpose it can be erased at will. The magnetizing pulses are brief pulses of current in the magnetizing coil of a specially designed electro-magnet usually referred to as the input or recording head. The localized magnetic field produced by the current pulse magnetizes the recording medium whose properties determine the magnetic strength of the minute magnets produced. Successive current pulses of opposite polarity will produce magnets in the wire whose adjacent poles have the same sign. Likewise successive current pulses of the same polarity will produce magnets whose adjacent poles are unlike. When the magnetized wire is moved across the air gap of an output head similar in construction to the input head, pulses of voltage are generated in the output coil surrounding a

1 This work was partially supported by the Office of the Chief of Ordnance, Department of the Army. lamination. If the magnets are crowded too closely together in the wire, the output voltage pulses will be affected. However, in order to conserve space it is necessary that the maximum number of pulses be recorded in each linear inch of the medium without the output pulses losing distinctness or objectionably blending with the preceding or succeeding pulse. The term "pulse packing" will be used to designate the number of magnets per unit length of the recording medium.

The maximum practicable pulse packing is limited by the interference between successive output pulses and by the signal-to-noise ratio for the output pulses. Since the factors that tend to increase the output voltage at the same time tend to increase the interaction between adjoining magnets, it is necessary to select as small an output voltage as is feasible and then to determine the greatest pulse packing for that voltage and the allowable interference.

Fortunately, in pulse recording as applied to electronic digital computers, distortion in the output pulses is not a critical factor, since the pulses generally operate trigger circuits. Moreover, the peak amplitude of the output pulse can vary considerably since clipping circuits are used between the output heads and the trigger circuits.

Investigations of the magnetizing current, pulse duration, and repetition rate as related to the amplitude and allowable interference of the output pulses on a wire recording medium have been made and are reported in this paper. A technique 
for visually examining the magnetized wire and determining the length of the magnetized section produced in the wire by the current pulses is also described.

\section{Input and Output Pulses}

Since it was desired to study not only the effects of amplitude and repetition rate of the recording pulses but also the effect of the duration of the peak current on the output pulses, recording pulses having an approximately rectangular shape were chosen for use in the experiments. Furthermore, since sequences of positive and negative current pulses are chosen for coding "words," rectangular pulses of both polarities are required. Each pulse of current in the magnetizing head produces a pair of poles or a minute magnet in the wire. The polarity of the current pulses will determine the direction of the magnet. Successive magnetizing pulses of opposite polarity produce a succession of short cylindrical magnets with adjacent poles of like polarity joined by a ferro-magnetic medium. If the duration of the magnetizing pulses is short compared to the speed of the wire, the elongation of the small magnet on the wire that results from the pulse duration may be neglected. Since the wire speed in these experiments was $12 \mathrm{in} . / \mathrm{sec}$, magnetizing pulses

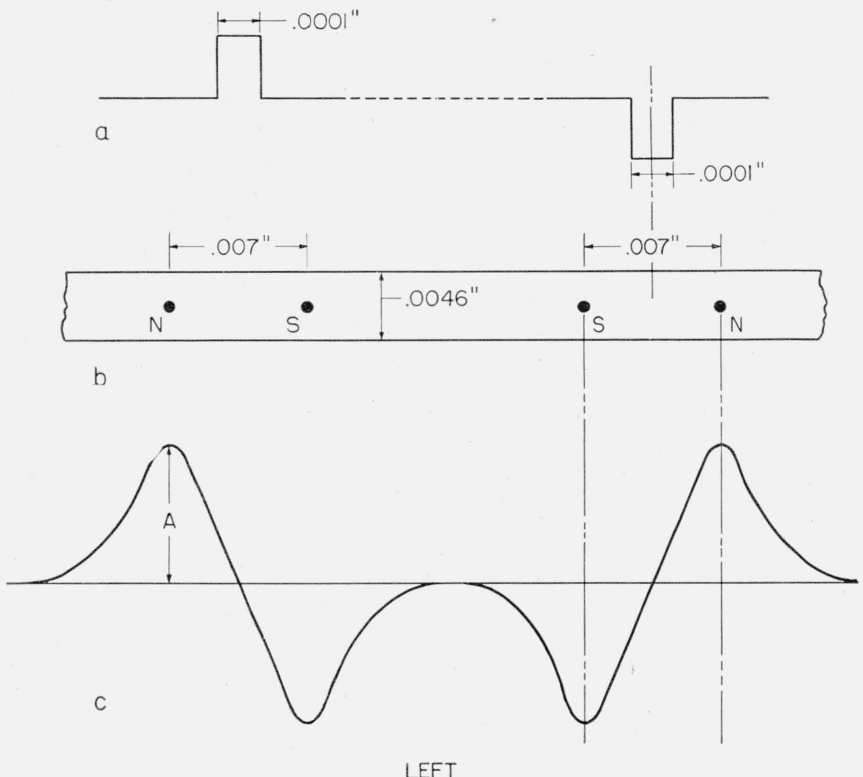

LEFT

of less than 20 microseconds duration lengthen the magnet less than 0.00024 in.

When a magnetized wire is drawn across the air gap of the output head, the magnets serve as a source of magnetizing force, and the resulting induction in the head links the output coil. Changes in flux linkages as the magnets move across the air gap induce voltages in the coil. As the magnets are packed closer and closer together in the wire, more effect is produced between the magnetic fields of adjoining magnets until sufficient distortion is produced in the magnetic fields to measurably change the shape of the output voltage pulses. Also, the overlapping of magnetic fields prevents the amplitude of the output voltage from becoming zero between successive magnets. The ratio of the minimum output voltage between magnets to the maximum output voltage is defined in this report as the interference ratio.

\section{Interference Ratio}

In $a$, left side of figure 1, are shown two input current pulses of opposite polarity. These two pulses produce two magnets in the wire as shown in $b$. These magnets produce two pairs of readback pulses as shown in $c$. For the case shown in the left side of figure 1 the repetition rate of the input pulses is low enough so that the output

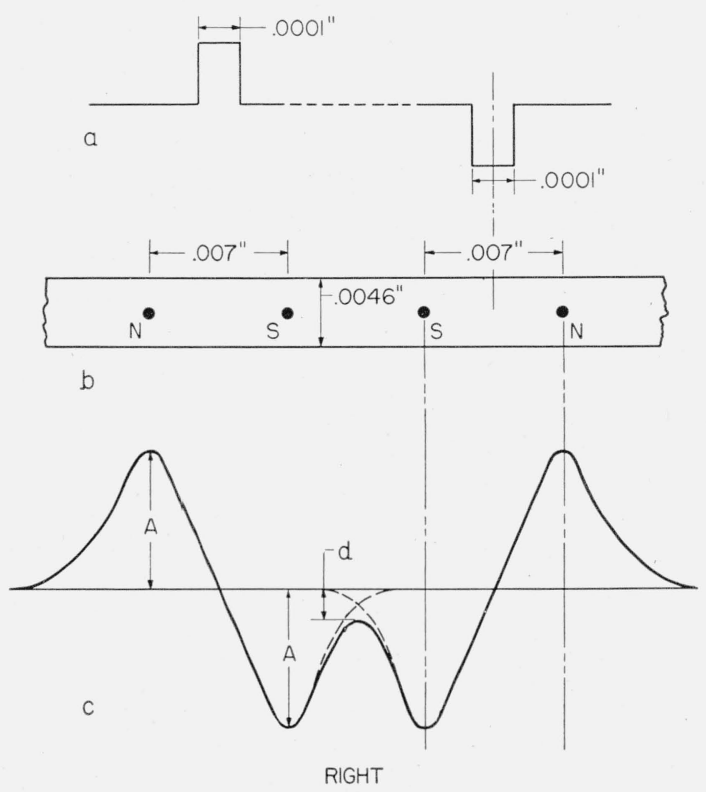

Figure 1. Magnetization of recording wire by rectangular current pulses and the output voltage pulses.

Left: a, Magnetizing pulses duration, 10 microseconds; amplitude, $40 \mathrm{ma}$. b, Magnets in recording wire. c, Output voltage pulses interference ratio $=0$. Right: a, Magnetizing pulses duration, 10 microseconds; amplitude, $40 \mathrm{ma}$. b, Magnets in recording wire. c, Output voltage pulses interference ratio $=d / A$. 
voltage (c) drops to zero between successive magnets. In the right side of figure 1 the repetition rate is sufficiently high to prevent the amplitude of the read-back voltage in $c$ to return to zero between magnets. In this figure the interference ratio is shown as $d / A$. It is obvious that the interference ratio is zero for successive pulses of the same polarity. An interference ratio of 0.1 (or $10 \%$ ) was chosen as the criterion of satisfactory pulse packing. Consequently $d$ in right side of figure 1 should not exceed 10 percent of $A$.

It should be noted from figure 1 that whereas the magnetizing pulse has a duration of 10 microseconds corresponding to a length of only 0.00012 in. on the wire at a wire speed of $12 \mathrm{in}$./sec. it produces a magnet several thousandths of an inch long in the wire. Later in this report this will be explained as resulting from the extended magnetic coupling between the wire and head.

\section{Apparatus}

\section{Loop Tester}

In order to study the effects of input pulse parameters on pulse packing, apparatus was constructed that requires a single continuous loop of recording wire approximately $8 \mathrm{ft}$. long. The advantages of a loop tester over apparatus using reels include simplification of the driving mechanism and the elimination of reeling difficulties.

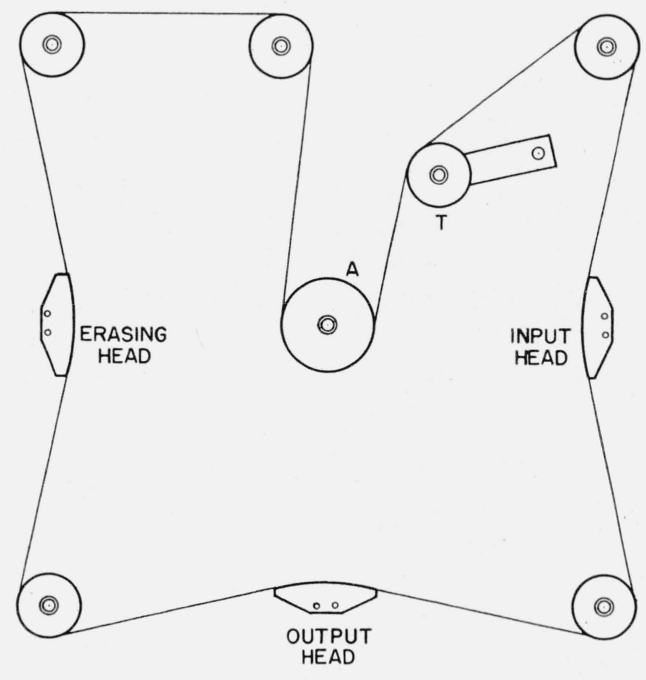

Figure 2. Loop tester.
The use of a short length of wire also decreases the probability of variations in output pulses arising from nonuniform magnetic characteristics that can occur in long lengths of recording wire. Separate input, output, and erasing heads were used. These heads were spaced sufficiently far apart to prevent noticeable electro-magnetic interaction between the heads. Figure 2 shows the top view of the loop tester. The driving force is supplied to the wire loop through the driving pulley, $A$, by a multipole phonograph motor mounted beneath and direct-coupled to the driving pulley. The wire is positioned by five grooved pulleys. The take-up pulley, $T$, serves to adjust the tension in the wire so that good contact is possible between the heads and the wire.

\section{Wires and Heads}

Two types of wire were tested, solid stainless steel and Co-Ni plated wire. ${ }^{2}$ The stainless steel wire is a solid metal wire specially cold worked in the drawing process in order to produce the magnetic properties suitable for magnetic recording wire. The stainless steel wire generally used has a diameter of $0.004 \mathrm{in}$. The plated wire consists of a magnetic coating of cobalt-nickel alloy plated on nonferrous material such as brass or phosphor bronze. The magnetic coating is generally 0.0003 in. thick, and the nonmagnetic wire is 0.004 in. in diameter. Thus the plated wire is $0.0046 \mathrm{in}$. in diameter compared with 0.004 in. for the stainless steel wire (see fig. 3). The values obtained for the intrinsic coercive force $\left(H_{c i}\right)$ and the residual induction $\left(B_{r}\right)$ of the wires used in this investigation are given in the following tabulation.

\begin{tabular}{|c|c|c|}
\hline Type of wire & $H_{c i}$ & $B_{r}$ \\
\hline $\begin{array}{l}\text { Stainless steel } \ldots \ldots \\
\text { Plated wire }\end{array}$ & $\begin{array}{l}\text { Oersted } \\
150 \\
220\end{array}$ & $\begin{array}{l}\text { Gauss } \\
3,700 \\
9,700\end{array}$ \\
\hline
\end{tabular}

These values were obtained from a maximum magneti ing force of 1,000 oersteds.

The magnetization of the wire is longitudinal along the wire parallel to the direction of motion. Transverse recording cannot be used, since twist-

${ }^{2}$ Frequency response of magnetic recording by Otto Kornei, Electronics, Vol. 20, No. 8, pp. 124 to 123 (August 1947). 


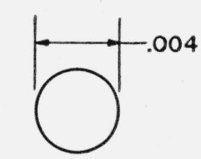

STAINLESS STEEL

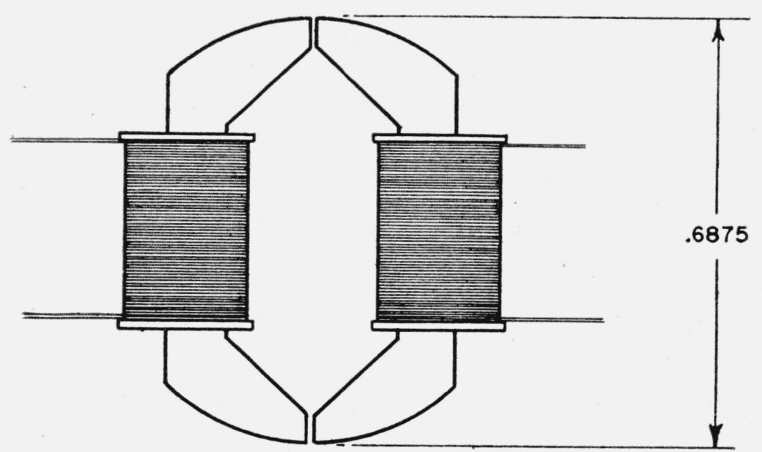

Figure 3. Two types of magnetic recording wire and a magnetizing head.

ing or rotation of a wire about its longitudinal axis during spooling could reverse the polarity of the magnet as read back by the output head. This change in polarity could result in errors in the numerical values of the coded numbers.

The magnetic heads were not especially designed and constructed for pulse recording but are commercially available sound recording heads. Their construction is shown in figure 3 . Each head consists of a core of two molybdenum permalloy laminations, $0.014 \mathrm{in}$. thick, with a coil of 900 turns surrounding each lamination. The gaps between the laminations are approximately 0.001 in. long. In addition to the gaps between the laminations, there is also an effective air gap between the recording wire and the laminations. Even small spatial variations in this gap between the wire and head can produce appreciable changes

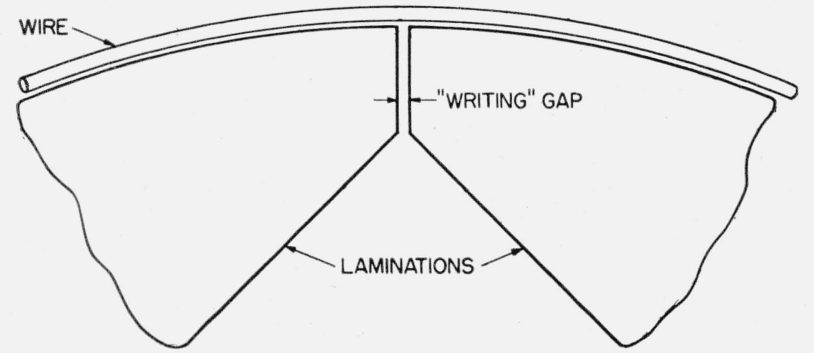

Figure 4. Enlarged view of the region near a "writing" gap of a magnetizing head. in the size and shape of the output pulses. An enlarged view of the "writing" gap between the lamination and the smaller effective air gap between the wire and head is shown in figure 4 . The wire and the head are magnetically coupled for some distance each side of the writing gap by the fringing flux. This may account for the fact that the magnets produced in the wire are several times as long as the gap.

\section{Recording Circuit}

As mentioned above, the magnetizing heads used have two coils, one on each lamination. This allows a simple circuit to be used for producing successive magnetizing pulses that produce fields having opposite directions.

A schematic diagram of the circuit and apparatus used for supplying input pulses of opposite polarity to the recording head is shown in figure

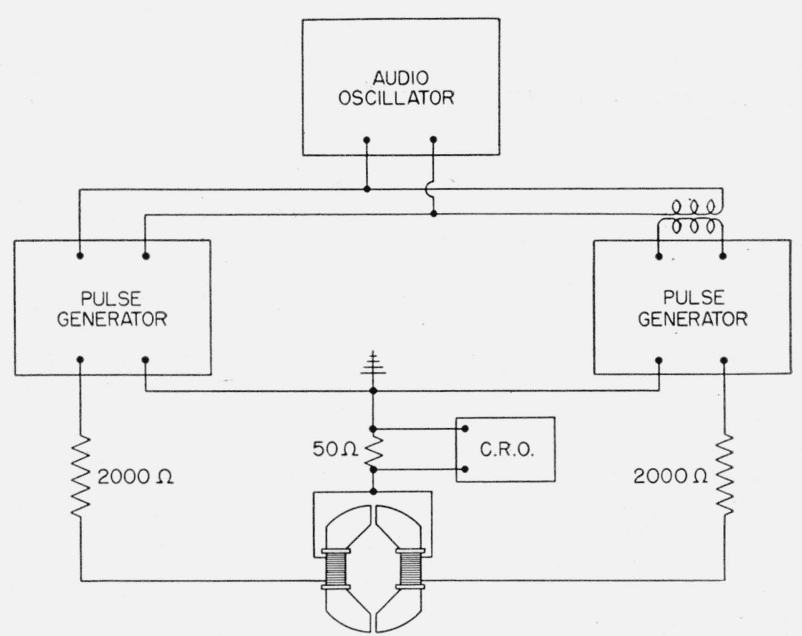

Figure 5. Recording circuit for rectangular input pulses.

5. In this figure is shown an audio oscillator supplying the input of two pulse generators. One pulse generator is fed directly from the oscil. lator, and the other pulse generator is fed through a transformer. The transformer is used to shift the phase of the input voltage to this pulse generator by approximately $180^{\circ}$ from the input voltage of the direct-fed pulse generator. The ground sides of the generators are connected together at one terminal of a 50 -ohm resistor. The resistor is then connected to the common side of the magnetizing coils. The other terminal of each generator is connected to a magnetizing coil through a resistor of $2,000 \mathrm{ohms}$. The 2,000-ohm 
resistors were found necessary to obtain a satisfactory time constant for the circuit. With the above circuit constants, the magnetizing current pulse as observed across the 50-ohm resistor by the cathode ray oscillograph is practically rectangular. The amplitude and shape of the current pulses, as well as the time relations of the pulses from the two generators can be observed on the cathode ray oscilloscope. This oscilloscope is calibrated by means of a sine wave, so that peak values of current can easily be determined.

The pulse repetition rate is controlled by the oscillator and the pulse duration, amplitude, and polarity are determined by the dial settings on the pulse generators. By the use of the apparatus and circuit described above, the recording wire can be magnetized by current pulses of alternate polarities or the same polarity. Thus studies can be made of the effect of pulse amplitude, duration, and repetition rate on the interference ratio.

\section{Output Apparatus}

The current pulses in the magnetizing coils are recorded in the wire as minute magnets. As the magnetized wire is moved across the air gap of the output head, varying induction is produced in the permalloy core; the variations in induction through the coil generate voltages at the coil terminals having peak values up to 5 or $6 \mathrm{mv}$. This small alternating voltage is amplified and then observed on a calibrated cathode ray oscilloscope. A battery-operated resistance-capacity coupled preamplifier is connected between the output head and the amplifier of the cathode ray oscilloscope. On the screen of the oscilloscope, the values of $d$ and $A$ are read for different values of pulse duration, amplitude, and repetition rate. The oscilloscope is calibrated to read peak voltages so that the output voltage as well as the interference ratio can be obtained from the data. Since stationary synchronized patterns are produced on the oscilloscope, the reading of $d$ and $A$ can be made fairly accurately.

\section{Erasing Circuit}

A head similar in construction to the input and output heads is used as the erasing head. It was experimentally found that when using a wire speed of $12 \mathrm{in} . / \mathrm{sec}$, an erasing current of $17 \mathrm{ma}$ peak to peak at a frequency of $20 \mathrm{kc} / \mathrm{sec}$ is suitable for demagnetizing the wire. This current is supplied from a power amplifier connected to the output of an oscillator. During tests with the loop tester the erasing circuit was continuously energized so that the pulses are first recorded, then read back and finally erased.

\section{Results}

The over-all resolution achieved in a pulse recorder depends both upon the length of the magnets produced in a given wire by the input head and upon the resulting pulses read back at the output head. In sound recording it is well known that the mechanical and magnetic requirements of the output head are much more critical than for the input head. Consequently for this investigation all available heads were successively tried as the output head in the loop tester, and the head that gave the smallest interference ratio for a given magnetizing current, pulse duration, and repetition rate was selected for the output head. It was found that among the 18 heads tried in the output position there was a spread of approximately 50 percent in the pulse packing for the same interference ratio. This indicates that the data presented on pulse packing can be considered only as a comparison between wires tested or as relative effects of certain parameters on the same wire and may not represent the greatest packing possible.

The data are also presented in most cases with the peak input current as a variable, whereas for comparison purposes it would be more logical to state the variable as current-turns. However since each magnetizing coil has 900 turns, ampereturns can be obtained by multiplying the current in milliamperes by 0.9 .

Comparisons between the output peak voltages as a function of input peak currents for the two types of wire are shown in figure 6 . These curves indicate that the output voltage for an input pulse of 10 -microseconds duration is proportional to the peak input current until a peak current of approximately $20 \mathrm{ma}$ is supplied to the magnetizing coil. Then the curves tend to level off so that a large increase in input current causes only a slight increase in the output voltage. The repetition rate for a given pulse duration and peak input current has only a slight effect on the output 


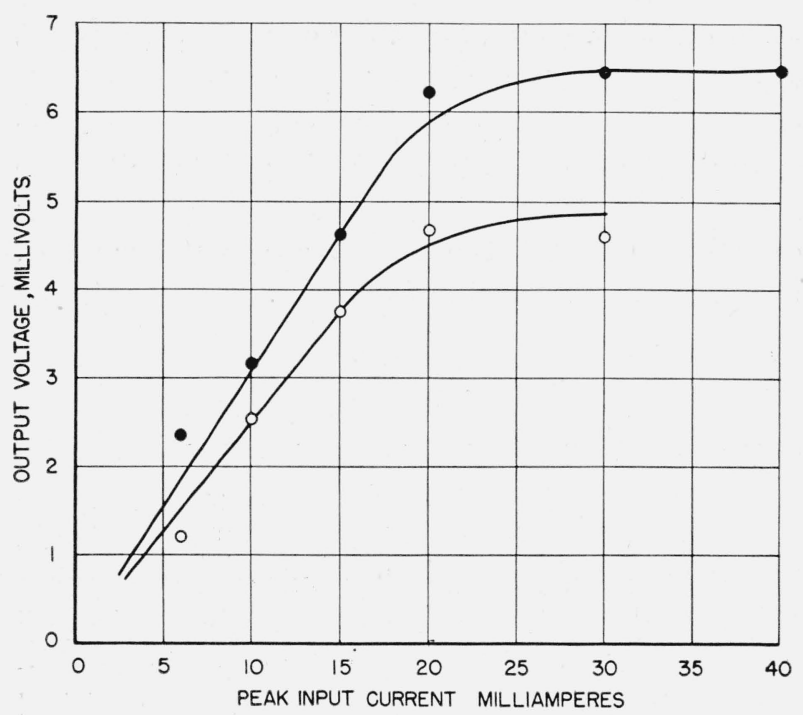

FIGURE 6. Variation of peak voltage of output pulses with magnetizing current.

๑, Plated wire; $\bigcirc$, stainless steel wire; Wire speed, 12 in./sec; pulse duration 10 microseconds.

voltage. For example, the output voltage for the plated wire remained constant at $4.65 \mathrm{mv}$ when the pulse repetition rate was varied from 50 to 117 pulses per inch.

Figure 7 shows the effect on output peak voltage of increasing the duration of the input pulse. In

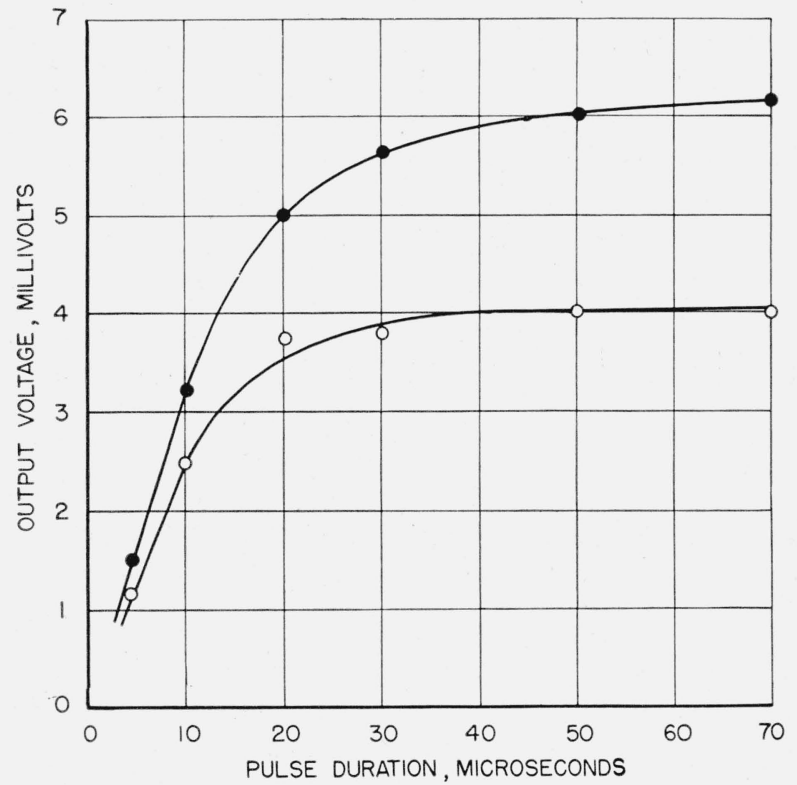

FIGURE 7. Variation of peak voltage of output pulses with duration of input pulses.

, Plated wire; $\bigcirc$, stainless steel wire; Wire speed 12 in./sec; peak input current $10 \mathrm{ma}$. general the output voltage is proportional to pulse duration for short pulses. As the pulse duration is increased, smaller and smaller relative increases occur in output voltage until the curves apparently level off. ${ }^{3}$ Under similar conditions the plated wire has a greater signal output for the same input.

Typical curves for the effect of peak input current on the interference ratio, using plated wire as a recording medium, are shown in figure 8 .

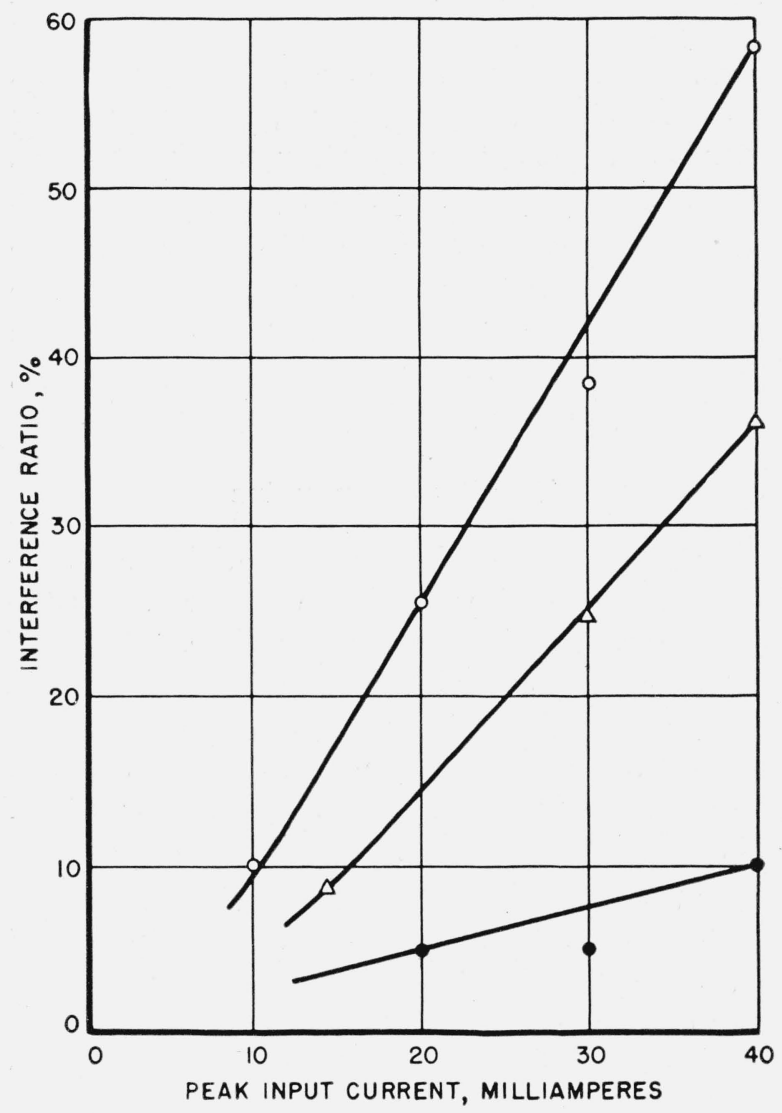

FIgURE 8. Variation of interference ratio with peak input current.

In this figure ppi is the abbreviation for pulses per inch. Plated wire; pulse duration 5 microseconds; wire speed $12 \mathrm{in./sec.} \bigcirc, 100 \mathrm{ppi} ; \triangle, 80 \mathrm{ppi}$; , 60 ppi.

These curves show that for an input pulse duration of 5 microseconds, and a repetition rate of 60 pulses per inch, the input current may be as large as $40 \mathrm{ma}$ before the interference ratio exceeds 10

${ }^{3}$ L. Cahn of the Bureau's Electronics Computers Section has investigated the storage of pulses whose durations are between 50 microseconds and 40 milliseconds, using plated wire and a wire speed of 2 in./sec. His results show that increasing the pulse duration from 50 microseconds to 5 milliseconds increases the output peak voltage by a factor of two. Further increases in pulse duration produce only small increases in output voltage. 
percent. However, if the repetition rate is increased to 80 pulses per inch, 15 ma would be the maximum permissible input current to keep the interference ratio as low as 10 percent. If the repetition rate is increased to 100 pulses per inch, the peak current cannot exceed 10 ma or less if the interference ratio is not to exceed 10 percent.

Figure 9 shows the dependence of the interference ratio on the pulse duration when a peak input current of $15 \mathrm{ma}$ is used. An increase in pulse duration results in an increase in the interference ratio.

Typical design curves can be assembled from the data already presented if two parameters (e. g. pulse duration and interference ratio) are fixed. On the basis of previous experience a pulse duration of 10 microseconds was chosen. Also it was tentatively decided to use an interference ratio of 10 percent as a criterion of suitable performance. Then the relationship between the pulse packing and the peak input current for the two types of wire is represented by the curves in figure 10 . From these curves it may be seen that a pulse packing of 60 per inch may be obtained with stainless steel wire for a peak input current of 6 to 7 ma, whereas for the plated wire as much as 23 or 24 ma could be used and a much greater output

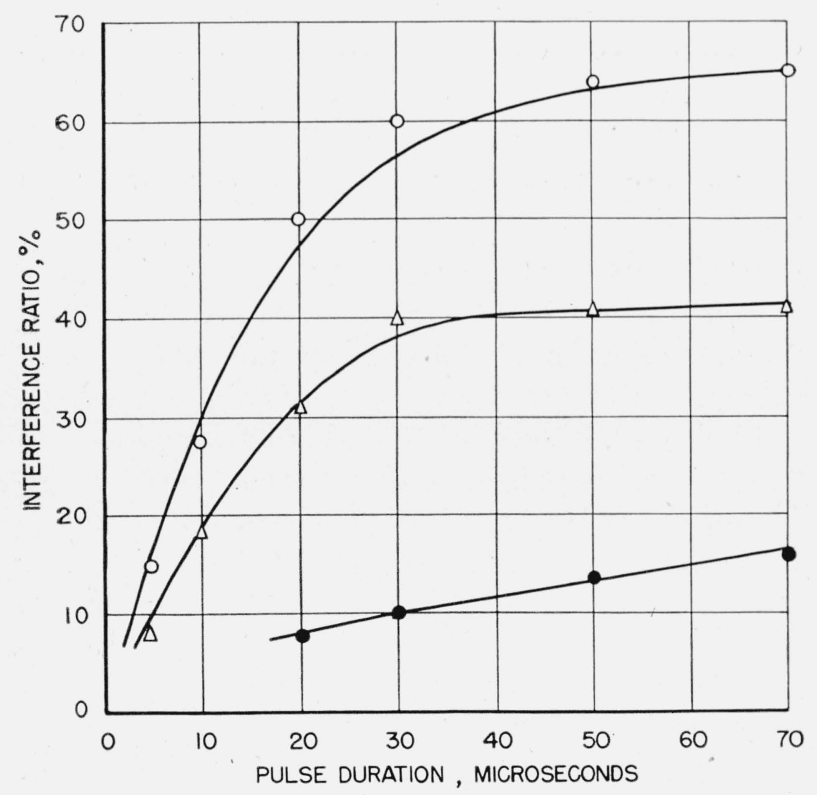

Figure 9. Variation of interference ratio with duration of input pulses.

In this figure ppi is the abbreviation for pulses per inch. Plated wire; peak current $15 \mathrm{ma}$; wire speed, $12 \mathrm{in}$./se. $\bigcirc, 100 \mathrm{ppi} \triangle \triangle, 80 \mathrm{ppi}$;, $60 \mathrm{ppi}$.

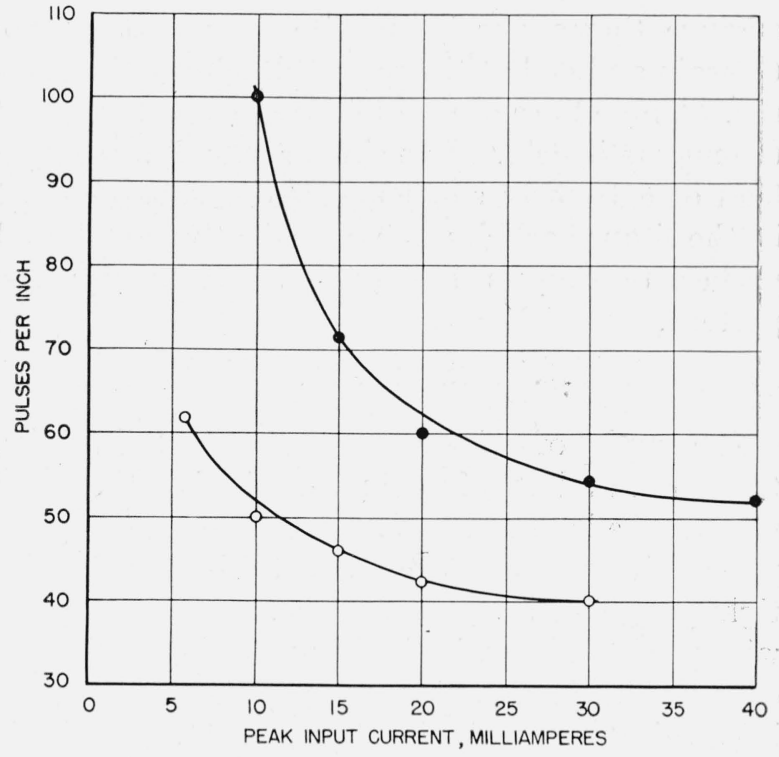

Figure 10. Relation between pulse packing and peak input current for two types of wire.

Pulse duration, 10 microseconds; interference ratio, 10 percent; wire speed, 12 in./sec. P. Plated wire; $\bigcirc$, stainless steel.

signal strength obtained, as is shown in figure 6 . If more than 100 pulses per inch are desired, this packing could be obtained with the plated wire when less than $10 \mathrm{ma}$ is used in the input head.

\section{Magnetic Patterns}

Studies of the magnetizing current, pulse duration, and repetition rate as related to the interference ratio gave useful data on the over-all performance of a pulse recorder. It is known, however, that the length of the air gap in the output or reproducing head has considerable effect on the high frequency response of magnetic recorders, and it was considered possible that the small magnets in the wire resulting from the current pulses may be "spread out" by the output head. Consequently it was very desirable to determine directly the length of the magnets on the wire. A wire retaining magnetically recorded pulses is similar to a succession of short cylindrical magnets laid in a line and connected end to end by a ferro-magnetic medium. Therefore if the magnetic field surrounding these minute magnets could be mapped and the pattern photographed, the length of the magnet could easily be determined. As is well known from elementary physics, iron filings when scattered about a magnet are not only attracted to the magnet but also 
arrange themselves in definite lines that appear to begin and end at the so-called poles of the magnet. These lines of iron filings are said to "map" the magnetic field. In a similar way the magnetic field of extremely small magnets can be mapped if the iron particles are sufficiently small and proper techniques are used in producing the pattern.

\section{Pattern Formation}

A sample of wire approximately 3 in. long, taken from a loop of wire magnetized in the usual way on the loop tester is mounted on a clean glass plate with sufficient tension to hold the wire tightly against the glass surface. The glass plate with the attached wire is then placed on the stage of a standard Bausch and Lomb Contour Measuring Projector. Referring to figure 11, the wire is shown at (5). The wire is illuminated by an optical system consisting of a tungsten arc lamp (1), a pair of achromatic condensers (2), and a mirror (3). The image of the wire is projected by a lens, roof prism, and mirror system onto a vertical screen. The vertical screen is an 8- by 10-in. ground glass plate upon which the magnified image of the wire is focused.

The fine magnetic powder used to delineate the external magnetic field is composed of carbonyl iron particles having an average diameter of approximately 0.0001 in. The carbonyl iron particles are suspended in an acid-free oil having an SAE viscosity rating of 10 . The proportion of iron particles to oil is not critical but is varied according to the density of pattern that is desired.

Referring again to figure 11, a drop of ironparticle suspension is placed on the wire at (5). The iron particles settle at a rate suitable to give a definite and distinct pattern of the external field surrounding the wire.

The particles in the strongest field (near the wire) are attracted and adhere to the magnetized portion of the wire and build up a pattern in a few minutes that extends out a distance approximately equal to one-half to the full diameter of the wire. The portion of the pattern than is produced on the glass plate takes longer for the settling to be completed since the magnetic forces acting there are much smaller. Usually a satisfactory pattern will be produced in approximately $30 \mathrm{~min}$. After the pattern is formed, the ground glass plate in the contour measuring projector is

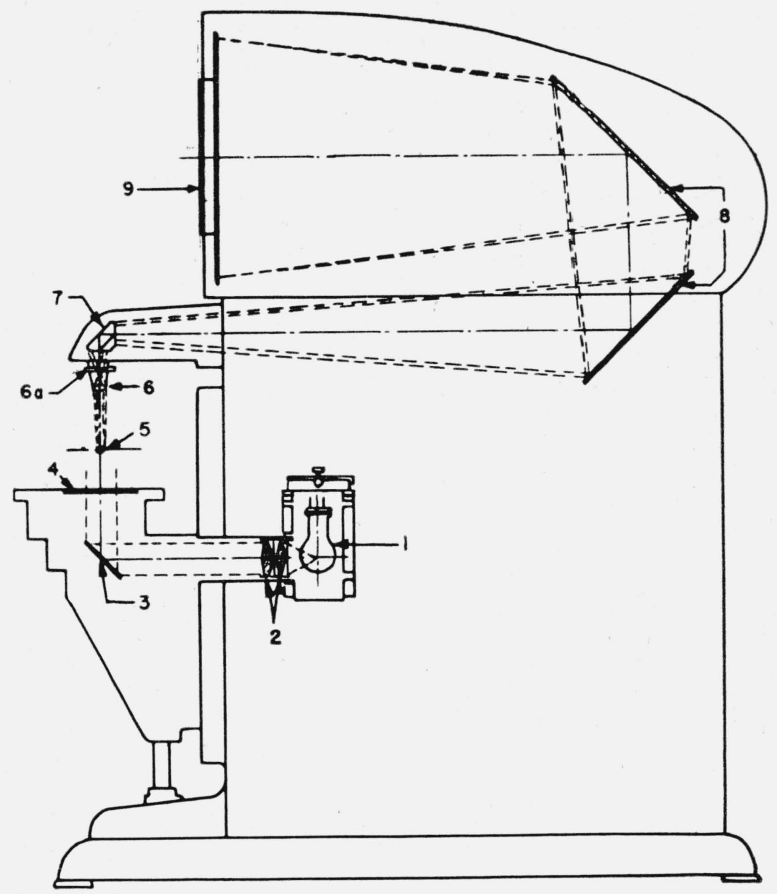

Figure 11. Modified Bausch and Lomb Contour Measuring Projector.

1, Tungsten arc lamp; 2, achromatic condensers; 3 , mirror; 4, stage; 5, magnetized wire; 6 , lens; 6 a, camera shutter; 7 , roof prism; 8 , mirrors; 9 , viewing screen.

replaced with film and the pattern photographed. The exposure time is controlled by means of a camera shutter $(6 \mathrm{~A})$ built into the lens mounting.

\section{Magnetic Field Patterns}

Figure 12 illustrates the type of pattern obtained by this method for plated wire $0.0046 \mathrm{in}$. in diameter, erased at a frequency of $20 \mathrm{kc} / \mathrm{sec}$ and recorded at a wire speed of $12 \mathrm{in} . / \mathrm{sec}$. The total magnification is 150 diameters unless otherwise stated.

Figure 12, A shows three magnets produced in the wire with pulses of 40 ma peak current, 70microseconds duration and a pulse-repetition rate of 60 pulses per inch. With only a small number of carbonyl iron particles in the field, a clear and distinct pattern is obtained, which allows measurement of the effective length of the magnets. For comparative purposes the effective length of the magnèt is taken as the distance between the poles. Figure 12, B is a photograph of the same pulses as in figure 12, A except that more carbonyl iron particles have been added and the extended field is clearly shown. The successive magnetizing 

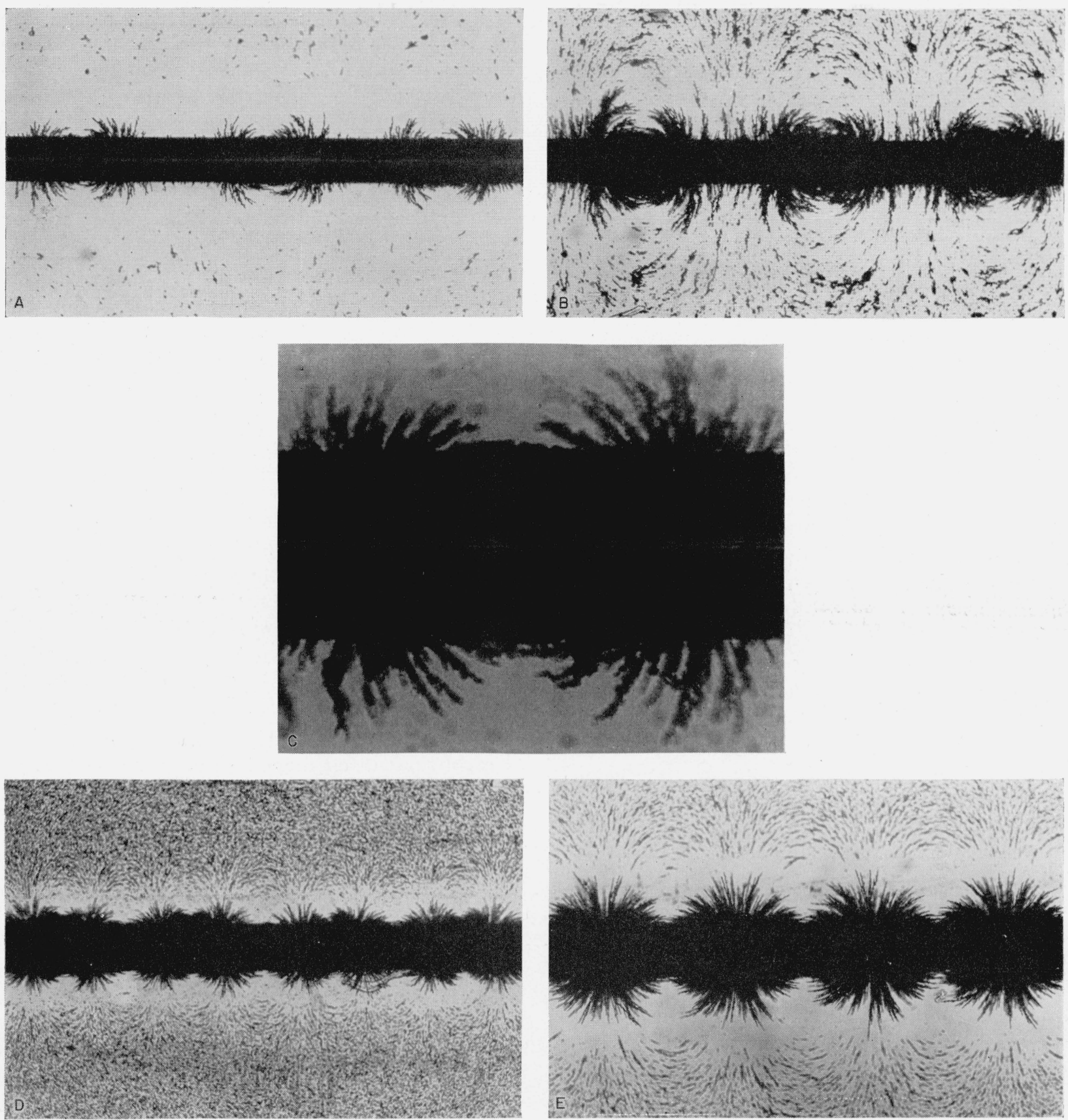

Figure 12.

A, Magnets produced by three pulses of opposite polarity; peak current, $40 \mathrm{ma}$; pulses per inch, 60 ; pulse duration, 70 microseconds. B, Same as A but showing the extended field. C, Pulse from A, total magnification 640 diameters. D, Magnets produced by four pulses of the same polarity; peak current, $40 \mathrm{ma}$; pulses per inch, 70; pulse duration, 10 microseconds. E, Magnets produced by a sine wave current; peak current, 8.5 ma; cycles per inch, 35 . 
pulses were of opposite polarity, so that neighboring poles of successive magnets are of the same kind and result in characteristic patterns as shown in the photograph.

A further enlargement of one of the pulses in figure $12, \mathrm{~A}$ is shown in figure 12, C. This photograph was made with an enlargement of 640 diameters.

Figure 12, D shows four magnets produced by four pulses of the same relative polarity. These pulses had a peak current of 40 ma, a duration of 10 microseconds, and a repetition rate of 70 per inch. Neighboring poles of adjacent magnets are of opposite sign.

The type of pattern obtained when the wire is magnetized with a sine wave current is shown in figure $12, \mathrm{E}$. The peak value of current in 1,800 turns was $8.5 \mathrm{ma}, 35$ cycles per inch being recorded. At a wire speed of 24 in./per sec (frequently used in magnetic recording) this would correspond to a recorded tone of $840 \mathrm{c} / \mathrm{s}$. If each sine wave is considered as two pulses of opposite polarity, each of the four poles shown in the photograph is seen to be a pair of poles of the same sign.

In comparing the results obtained by the visual method with the data on pulse packing, it must be borne in mind that the output pulse voltage begins before the leading pole of the magnet reaches the writing gap and continues after the trailing pole has left the gap. Measurements made on the photograph of figure 12, A and others, indicate that the average distance between poles is of the order of $0.008 \mathrm{in}$. for magnetizing pulses having a peak value of $40 \mathrm{ma}$ and a duration of 70 microseconds. These photographs also show that the external field of the minute magnets is appreciable for several thousandths of an inch beyond each pole. Oscillograph records obtained with the loop tester using similar pulse parameters for recording, indicate that the duration of the voltage of the output pulse corresponds to the time required for the wire to travel between 0.016 and $0.020 \mathrm{in}$., which is between 2 and $2 \frac{1}{2}$ times the distance between the poles of the magnet as indicated visually.

\section{Conclusions}

A method and apparatus are described for determining the relative pulse packing of different types of magnetic recording wire used for pulse storage. Typical curves are presented to show the influence of the amplitude, duration, and repetition rate of the magnetizing pulses on the pulse packing in two types of recording wire.

A powder pattern method is described for visually observing the length of the magnets produced by the magnetizing pulses. The data obtained by this method verify the pulse-packing data obtained by the interference ratio method.

Using an interference ratio of 0.1 as a criterion, it is possible to pack approximately 100 pulses per inch in the plated wire, when the input pulses have a length of 0.0001 in. (i. e. 10 microseconds) and a peak current of less than 10 ma.

From a study of the photographs (fig. 12) it is concluded that magnets whose average length is $0.008 \mathrm{in}$. are produced in plated wire $0.0046 \mathrm{in}$. in diameter by magnetizing pulses of 40 ma through 900 turns (i. e. 36-amp turns), if the duration of the input magnetizing pulse is 70 microseconds, and the wire speed is $12 \mathrm{in} . / \mathrm{sec}$. If the input pulse parameters are the same except that the duration is decreased to 10 microseconds, the average length of the magnet is decreased by $0.001 \mathrm{in}$.

Washington, August 17, 1949. 